\title{
Analysis of stationary random vibrating systems using smooth decomposition
}

\author{
Sergio Bellizzi ${ }^{\mathrm{a}, *}$ and Rubens Sampaio ${ }^{\mathrm{b}}$ \\ ${ }^{\mathrm{a}}$ LMA, CNRS, Aix-Marseille University, Marseille, France \\ ${ }^{\mathrm{b}}$ Department of Mechanical Engineering, PUC-Rio, Rio de Janeiro, Brazil
}

Received 5 July 2012

Revised 4 November 2012

Accepted 9 November 2012

\begin{abstract}
A modified Karhunen-Loève Decomposition/Proper Orthogonal Decomposition method, named Smooth Decomposition (SD) (also named smooth Karhunen-Loève decomposition), was recently introduced to analyze stationary random signal. It is based on a generalized eigenproblem defined from the covariance matrix of the random process and the covariance matrix of the associated time-derivative random process. The SD appears to be an interesting tool in terms of modal analysis. In this paper, the SD will be described in case of stationary random processes and extended also to stationary random fields. The main properties will be discussed and illustrated on a randomly excited clamped-free beam.
\end{abstract}

Keywords: Karhunen-Loève decomposition, random vibration, random fields, modal analysis

\section{Introduction}

The Karhunen-Loève Decomposition (KLD) also known as Proper Orthogional Decomposition (POD) has been extensively used as a tool for analyzing random processes and random fields. The KLD reveals some coherent structures which have been advantageously used in different domains as, for example, the stochastic finite elements method, the simulation of random fields, the dynamical analysis of nonlinear systems [9], and in the construction of reduced order models $[1,4,7]$.

Recently, a new multivariable data analysis method originally called Smooth Orthogonal Decomposition (SOD) has been proposed in [5]. The SOD can be viewed as a tool to extract structures from a maximization problem associated to a scalar time series of measurement but subject to a minimization constraint acting on the associated time derivative of the time series. The constraint ensures that the time variation of the component associated to the structures is as small as possible. The SOD can be used to extract normal modes and natural frequencies of multi-degree-of-freedom vibration systems. Free and forced sinusoidal responses have been considered in [5] and randomly excited systems have been analyzed in [6]. The Smooth Orthogonal Decomposition has been formulated in term of a smooth Karhunen-Loève decomposition to analyze time continuous random processes in [3]. The decomposition was extended to the non-stationary case in [11]. The smooth Karhunen-Loève decomposition is obtained solving a generalized eigenproblem defined from the covariance matrix of the random field and the covariance matrix of its time derivative. These two matrices are output-only data dependent. In this paper, as in [11], the smooth Karhunen-Loève decomposition will be renamed as Smooth Decomposition (SD) since it does not have the properties of a Karhunen-Loève decomposition.

\footnotetext{
* Corresponding author: Sergio Bellizzi, LMA, CNRS, UPR 7051, Centrale Marseille, Aix-Marseille University, F-13420 Marseille Cedex 20, France. E-mail: bellizzi@lma.cnrs-mrs.fr.
} 
This paper presents and discusses the SD for continuous processes in time and for continuous processes in space and time, classically named random fields. The techniques used so far to show the relation between the smooth modes and the normal modes rely on the possibility to write both problems, the covariance definition of smooth modes and the eigenvalue problem defining the normal modes, in matrix form and to reduce one form to the other under convenient hypothesis. This technique can be applied only because the number of degrees of freedom is finite. This technique cannot be used in the random-field case, that is, to write in a global form the covariance operator and the eigenvalue problem for continuous systems. The resulting SD characteristics are discussed in the case of vibrating clamped-free beam subjected to white-noise excitation, in terms of the normal modes. The results are also compared to results obtained from a KLD analysis.

\section{Smooth orthogonal decomposition of a $\mathbb{R}^{n}$-valued random process}

Without loss of generality, we will assume that $\{\mathbf{U}(t), t \in \mathbb{R}\}$ is a zero-mean random process and that $\mathbf{R}_{\mathbf{U}}$ and $\mathbf{R}_{\dot{U}}$ are symmetric positive definite.

\subsection{Decomposition principle}

The Smooth Decomposition (SD) of $\{\mathbf{U}(t), t \in \mathbb{R}\}$ aims at obtaining the most characteristic constant vectors, or structures, $\Gamma$ in the sense that they maximize the ratio of the ensemble average of the inner product between $\mathbf{U}(t)$ and $\Gamma$ and the inner product between $\dot{\mathbf{U}}(t)$ and $\Gamma$

$$
\max _{\Gamma \in \mathbb{R}^{n}} \frac{\left.\mathbb{E}(<\mathbf{U}(t), \Gamma)>^{2}\right)}{\mathbb{E}\left(<\dot{\mathbf{U}}(t), \Gamma>^{2}\right)}
$$

where $<,>$ denotes the inner product in $\mathbb{R}^{n}$.

Due to the stationary property, the optimization problem Eq. (1) can be rewritten as

$$
\max _{\Gamma \in \mathbb{R}^{n}} \frac{\Gamma^{T} \mathbf{R}_{\mathbf{U}} \Gamma}{\Gamma^{T} \mathbf{R}_{\dot{U}} \Gamma}
$$

showing that the cost function depends on the covariance matrices of $\{\mathbf{U}(t), t \in \mathbb{R}\}$ and $\{\dot{\mathbf{U}}(t), t \in \mathbb{R}\}$. The vectors which yields local maximums are solutions of the following eigenproblem

$$
\mathbf{R}_{\mathbf{U}} \boldsymbol{\Gamma}_{k}=\sigma_{k} \mathbf{R}_{\dot{\mathbf{U}}} \boldsymbol{\Gamma}_{k}
$$

involving the covariance matrices $\mathbf{R}_{\mathbf{U}}$ and $\mathbf{R}_{\dot{U}}$. Assuming that the matrices are positive define, it is easy to show that the set of vectors $\boldsymbol{\Gamma}_{k}$ constitutes a basis which is $\mathbf{R}_{\mathbf{U}}$ and $\mathbf{R}_{\dot{\mathbf{U}}}$-orthogonal.

The SD of the random process $\{\mathbf{U}(t), t \in \mathbb{R}\}$ is then defined for this basis as

$$
\mathbf{U}(t)=\sum_{k=1}^{n} \xi_{k}(t) \boldsymbol{\Gamma}_{k}
$$

where the scalar random processes, $\xi_{k}(t)$, are given by

$$
\xi_{k}(t)=\frac{\boldsymbol{\Gamma}_{k}^{T} \mathbf{R}_{\mathbf{U}} \mathbf{U}(t)}{\boldsymbol{\Gamma}_{k}^{T} \mathbf{R}_{\mathbf{U}} \boldsymbol{\Gamma}_{k}}=\frac{\boldsymbol{\Gamma}_{k}^{T} \mathbf{R}_{\dot{\mathbf{U}}} \mathbf{U}(t)}{\boldsymbol{\Gamma}_{k}^{T} \mathbf{R}_{\dot{\mathbf{U}}} \boldsymbol{\Gamma}_{k}} .
$$

Note that the scalar processes $\left\{\xi_{k}(t), t \in \mathbb{R}\right\}$ can be defined from either $\mathbf{R}_{\mathbf{U}}$ or $\mathbf{R}_{\dot{\mathbf{U}}}$, that is, they do not depend on which one of these two covariance matrices is used in the computation. 
The following notation is used: the eigenvalues $\sigma_{k}$ are called the Smooth Values (SVs) $\left(\boldsymbol{\Sigma}=\operatorname{diag}\left(\sigma_{k}\right)\right)$, the eigenvectors $\boldsymbol{\Gamma}_{k}$ are called the Smooth Modes (SMs), and the scalar random processes $\left\{\xi_{k}(t)\right\}$ are called the Smooth Components (SCs). The following ordering $\sigma_{1} \geqslant \sigma_{2} \geqslant \ldots \geqslant \sigma_{n}$ will be used to sort the SMs.

The generalized eigenproblem Eq. (3) is a statistical version (for continuous-time random process), of the generalized eigenvalue problem introduced in [5] to characterize the SOD. This definition constitutes a major difference. In the definition Eq. (3) only the covariance matrices $\mathbf{R}_{\mathbf{U}}$ and $\mathbf{R}_{\dot{\mathbf{U}}}$ are used, no other operator is necessary. The idea comes from [3]. The results are, of course, similar to the ones presented in [5,6], but now, since one relies on the covariance matrices, one has a powerful computation tool, not available before.

The objective function used to define the SD differs significantly from that used to define the classical KarhunenLoève Decomposition [2]. Here the denominator of the objective function takes the covariance matrix of the timederivative process $\{\dot{\mathbf{U}}(t), t \in \mathbb{R}\}$ into account. This term assures that, for each mode, the time variation of the SC associated to the SM is as small as possible. This property justifies the term smooth used to name the decomposition.

\subsection{Some properties of the $S D$}

\subsubsection{Properties of SV, $S M$, and $S C$}

Assuming that the matrices $\mathbf{R}_{\mathbf{U}}$ and $\mathbf{R}_{\dot{\mathbf{U}}}$ are symmetric positive definite (which is generally the case), all the SVs (eigenvalues) $\nu_{k}$ are strictly positive and the set of the SMs $\boldsymbol{\Gamma}_{k}$ constitutes a basis which is orthogonal with respect to both covariance matrices $\mathbf{R}_{\mathbf{U}}$ and $\mathbf{R}_{\dot{\mathbf{U}}}$. Note that the $\mathrm{SM}$ are unique up to a scaling constant.

The scalar processes $\left\{\xi_{k}(t), t \in \mathbb{R}\right\}$ are correlated and the following result holds

$$
\mathbb{E}\left(\xi_{k}(t) \xi_{l}(t)\right)=\frac{\boldsymbol{\Gamma}_{k}^{T} \mathbf{R}_{\mathbf{U}} \mathbf{R}_{\mathbf{U}} \mathbf{R}_{\mathbf{U}} \boldsymbol{\Gamma}_{l}}{\boldsymbol{\Gamma}_{k}^{T} \mathbf{R}_{\mathbf{U}} \boldsymbol{\Gamma}_{k} \boldsymbol{\Gamma}_{l}^{T} \mathbf{R}_{\mathbf{U}} \boldsymbol{\Gamma}_{l}}=\frac{\boldsymbol{\Gamma}_{k}^{T} \mathbf{R}_{\dot{\mathbf{U}}} \mathbf{R}_{\mathbf{U}} \mathbf{R}_{\dot{\mathbf{U}}} \boldsymbol{\Gamma}_{l}}{\boldsymbol{\Gamma}_{k}^{T} \mathbf{R}_{\dot{\mathbf{U}}} \boldsymbol{\Gamma}_{k} \boldsymbol{\Gamma}_{l}^{T} \mathbf{R}_{\dot{\mathbf{U}}} \boldsymbol{\Gamma}_{l}}
$$

The SD does not define an optimum basis in terms of energy as the KLD approach does [2]. So, properly speaking, the SD is not a Karhunen-Loève decomposition. The introduction of regularity has then its drawbacks.

\subsubsection{Invariance with respect to linear transformations}

Some parameters defining the SD are invariant with respect to linear transformation. Consider the random process $\{\mathbf{V}(t), t \in \mathbb{R}\}$ defined as a linear transformation of $\{\mathbf{U}(t), t \in \mathbb{R}\}$ by

$$
\mathbf{V}(t)=\mathbf{A} \mathbf{U}(t)
$$

where $\mathbf{A}$ is a square invertible matrix.

Using the relationships $\mathbf{R}_{\mathbf{V}}=\mathbf{A} \mathbf{R}_{\mathbf{U}} \mathbf{A}^{T}$ and $\mathbf{R}_{\dot{\mathbf{V}}}=\mathbf{A} \mathbf{R}_{\dot{\mathbf{U}}} \mathbf{A}^{T}$ it can be shown that the SVs of $\{\mathbf{V}(t), t \in \mathbb{R}\}$ coincide with those of $\{\mathbf{U}(t), t \in \mathbb{R}\}$ and the sets of the SMs satisfy the condition

$$
\boldsymbol{\Gamma}_{k}(V)=\mathbf{A}^{-T} \boldsymbol{\Gamma}_{k}(U)
$$

where $\boldsymbol{\Gamma}_{k}(U)$ (respectively $\boldsymbol{\Gamma}_{k}(V)$ ) denotes a $\mathrm{SM}$ of $\{\mathbf{U}(t), t \in \mathbb{R}\}$ (respectively, of $\{\mathbf{V}(t), t \in \mathbb{R}\}$ ). Finally, following Eq. (5), the SCs are invariant with respect to the linear change of variables if and only if $\mathbf{A A}^{T}=\mathbf{I}$.

\subsection{Mechanical interpretation of $S D$}

The objective of this section is to find relationships between the parameters of the SD (SMs and SVs) and the normal modes (in terms of mode shapes and resonance frequencies) of a linear system with $n$ Degrees Of Freedom (DOF). Some relationships between the SMS and the mass matrix of the system will also be discussed. The equation of motion is written as

$$
\mathbf{M} \ddot{\mathbf{U}}(t)+\mathbf{C} \dot{\mathbf{U}}(t)+\mathbf{K U}(t)=\mathbf{F}(t)
$$

where $\{\mathbf{F}(t), t \in \mathbb{R}\}$, is a zero-mean white-noise random excitation (i.e., $\mathbf{R}_{F}(\tau)=\mathbb{E}\left(\mathbf{F}(t+\tau) \mathbf{F}^{T}(t)\right)=\mathbf{S}_{F} \delta(\tau)$, where the intensity $\mathbf{S}_{F}$ is a symmetric constant matrix). External excitation and damping terms have being considered in such a way to work with well defined covariance matrices, $\mathbf{R}_{\mathbf{U}}$ and $\mathbf{R}_{\dot{\mathbf{U}}}$.

The Normal Modes (NMs) associated to Eq. (9) are defined by the eigenproblem $\mathbf{K} \boldsymbol{\Phi}_{k}=\mathbf{M} \boldsymbol{\Phi}_{k} \omega_{k}^{2}$ with the normalization conditions $\boldsymbol{\Phi}^{T} \mathbf{M} \boldsymbol{\Phi}=\mathbf{I}$. Using the classical modal-displacement vector $\mathbf{Q}(t)$ defined by $\mathbf{U}(t)=$ $\mathbf{\Phi} \mathbf{Q}(t)$ the equation of motion Eq. (9) can be equivalently replaced by

$$
\ddot{\mathbf{Q}}(t)+\boldsymbol{\Theta} \dot{\mathbf{Q}}(t)+\boldsymbol{\Omega}^{2} \mathbf{Q}(t)=\boldsymbol{\Phi}^{T} \mathbf{F}(t)
$$

with $\Theta=\boldsymbol{\Phi}^{T} \mathbf{C} \boldsymbol{\Phi}$. 


\subsubsection{SD and modal analysis}

We will discuss here relationships between the SD of the steady-state (stationary) response of Eq. (9) and the modal parameters $\left(\omega_{i}^{2}, \boldsymbol{\Phi}_{i}\right)$.

If the damping is proportional (i.e., $\boldsymbol{\Phi}^{T} \mathbf{C} \boldsymbol{\Phi}=\operatorname{diag}\left(2 \tau_{i} \omega_{i}\right)$ is diagonal) and the matrix $\boldsymbol{\Phi}^{T} \mathbf{S}_{F} \boldsymbol{\Phi}$ is also diagonal (i.e., if the modal-excitation terms $\boldsymbol{\Phi}_{i}^{T} \mathbf{F}(t)$ in Eq. (10) are uncorrelated) then, as established in [3], the covariance matrices $\mathbf{R}_{\mathbf{Q}}$ and $\mathbf{R}_{\mathbf{Q}}$ of the stationary responses $\{\mathbf{Q}(t), t \in \mathbb{R}\}$ and $\{\dot{\mathbf{Q}}(t), t \in \mathbb{R}\}$ are diagonal. Hence the SM associated to the process $\{\mathbf{Q}(t), t \in \mathbb{R}\}$ are equal to the vector of the canonical basis of $\mathbb{R}^{n}$ and the SVs are given by the diagonal terms of the matrix $\mathbf{R}_{\mathrm{Q}}^{-1} \mathbf{R}_{\mathrm{Q}}$ which are given by $\left(\boldsymbol{\Omega}^{2}\right)^{-1}$. Now using the linear relation (see Section 2.2.2), we can easily deduce that the SVs of $\{\mathbf{U}(t), t \in \mathbb{R}\}$ coincide with the SVs of $\{\mathbf{Q}(t), t \in \mathbb{R}\}$ and that the SMs of $\{\mathbf{U}(t), t \in \mathbb{R}\}$ are given by $\boldsymbol{\Phi}^{-T}$. This relationship is determined up to a multiplicative constant.

To summarize, the SD has the following nice properties

$$
\boldsymbol{\Phi}=\boldsymbol{\Gamma}^{-T} \text { and } \boldsymbol{\Omega}^{2}=\boldsymbol{\Sigma}^{-1},
$$

the mode shapes of the normal modes are obtained by inverting the transpose of the SM matrix and the resonance frequencies are given by the inverse of the SVs (characteristic which is not easily to obtain from the KLD). This last relation justifies the ordering chosen to sort the SMs which is in line with ordering of the resonance frequencies.

It is interesting to note that, as indicated in [5], no assumption on the mass matrix $\mathrm{M}$ is needed to relate the NMs to the SMs whereas the KLMs coincide with the NMs only when the mass matrix is proportional to the identity matrix ( $\mathbf{M}=c \mathbf{I}$, where $c$ is any positive real number)[2]. Moreover, if $\mathbf{M}=c \mathbf{I}$, then the SMs coincide with the KLMs, hence, they both, of course, coincide with the LNMs.

\subsubsection{Influence of the mass inhomogeneity on the SM}

An interesting property of the SM is its sensitivity to the mass-inhomogeneity. Combining the following two equations, $\boldsymbol{\Gamma}=\boldsymbol{\Phi}^{-T}$ and $\boldsymbol{\Phi}^{T} \mathbf{M} \boldsymbol{\Phi}=\mathbf{I}$, the SM matrix reads as $\boldsymbol{\Gamma}=\mathbf{M} \boldsymbol{\Phi}$ and in case of mass-inhomogeneity, that is to say, when the mass matrix is diagonal i.e $\mathbf{M}=\operatorname{diag}(D)$ where $\boldsymbol{D}=\left(d_{1}, \ldots, d_{n}\right)^{T}$ then $\boldsymbol{\Gamma}_{k}=\boldsymbol{D}$. $\boldsymbol{\Phi}_{k}$ where “." denotes the element-by-element product. Each SM differs from a NM by a scaling vector factor given by the mass-inhomogeneity.

\section{Smooth decomposition of a random fields}

The objective of this section is to extent the SD to continuous in time and space random processes $\{\mathbf{u}(t, x)$, $\left.(t, x) \in \mathbb{R} \times \mathcal{D}_{x}\right\}$. The covariance functions $R_{\mathbf{u}}\left(0, x, x^{\prime}\right)$ and $R_{\dot{\mathbf{u}}}\left(\tau, x, x^{\prime}\right)$ will play the same role as the matrices $\mathbf{R}_{\mathbf{U}}$ and $\mathbf{R}_{\dot{\mathbf{U}}}$ in case of $\mathbb{R}^{n}$-valued random processes considered in the previous section.

\subsection{Definition and properties}

Recalling the eigenproblem Eq. (3) used to define the SD in the $\mathbb{R}^{n}$-valued case, the SD of a random field $\left\{\mathbf{u}(t, x),(t, x) \in \mathbb{R} \times \mathcal{D}_{x}\right\}$ can be defined as

$$
\mathbf{u}(t, x)=\sum_{k=1}^{+\infty} \zeta_{k}(t) \Gamma_{k}(x)
$$

where the deterministic functions $\Gamma_{k}$ solve the generalized eigenproblem

$$
\int_{\mathcal{D}_{x}} R_{\mathbf{u}}\left(0, x, x^{\prime}\right) \Gamma_{k}\left(x^{\prime}\right) d x^{\prime}=\nu_{k} \int_{\mathcal{D}_{x}} R_{\dot{\mathbf{u}}}\left(0, x, x^{\prime}\right) \Gamma_{k}\left(x^{\prime}\right) d x^{\prime}
$$

and the scalar random processes, $\zeta_{k}(t)$ are given by

$$
\zeta_{k}(t)=\frac{\int_{\mathcal{D}_{x}} \int_{\mathcal{D}_{x}} \Gamma_{k}^{T}(x) R_{\mathbf{u}}\left(0, x, x^{\prime}\right) u\left(t, x^{\prime}\right) d x d x^{\prime}}{c_{k k}}
$$


with

$$
c_{k l}=\int_{\mathcal{D}_{x}} \int_{\mathcal{D}_{x}} \Gamma_{k}^{T}(x) R_{\mathbf{u}}\left(0, x, x^{\prime}\right) \Gamma_{l}\left(x^{\prime}\right) d x d x^{\prime} .
$$

The eigenproblem Eq. (13) is a "space continuous" version of the generalized eigenvalue problem Eq. (3) to characterize the SD of a random process. The discussion on the existence and the properties of the solutions of this eigenproblem is out of the scope of this paper. We will assume here that the covariance functions $R_{\mathbf{u}}\left(0, x, x^{\prime}\right)$ and $R_{\dot{\mathbf{u}}}\left(0, x, x^{\prime}\right)$ as such that the eigenproblem Eq. (13) admits a countable number of eigenvalues $\nu_{1} \geqslant \nu_{2} \geqslant \ldots \geqslant$ $\nu_{k} \geqslant \ldots$, and that the set of associated normalized eigenfunctions, $\left(\Gamma_{k}\right)_{k \geqslant 1}$, constitute a basis of $L^{2}\left(\mathcal{D}_{x}, \mathbb{R}^{n}\right)$.

As in the discrete case (see Eq. (14)), the scalar processes $\left\{\zeta_{k}(t), t \in \mathbb{R}\right\}$ can be defined from either $R_{\mathbf{u}}\left(0, x, x^{\prime}\right)$ or $\left.R_{\dot{\mathbf{u}}} 0, x, x^{\prime}\right)$, that is, they do not depend on which one of this two covariance matrices is used.

As classical, the following notations will be used: the eigenvalues $\nu_{k}$ are called the Smooth Values (SVs), the eigenvector functions $\Gamma_{k}$ are called the Smooth Modes (SMs), and the scalar random processes $\left\{\zeta_{k}(t)\right\}$ are called the Smooth Components (SCs).

The SD differs significantly from the POD used for example in [1,4]. The SD are characterized from the "space" covariance matrices $R_{\mathbf{u}}\left(0, x, x^{\prime}\right)$ and $\left.R_{\dot{\mathbf{u}}} 0, x, x^{\prime}\right)$ whereas POD are derived from snapshots.

Finally, the properties of the parameters of the SD as described in Sections 2.2.1 and 2.2.2 can be extented to the random field case. All the SVs (eigenvalues) $\nu_{k}$ are strictly positive and the set of the functions $\Gamma_{k}$ (the SMs) constitutes a basis which is orthogonal with respect to both covariance matrix functions $R_{\mathbf{u}}\left(0, x, x^{\prime}\right)$ and $R_{\dot{\mathbf{u}}}\left(0, x, x^{\prime}\right)$. Here also, the scalar processes $\left\{\zeta_{k}(t), t \in \mathbb{R}\right\}$ are correlated. The SD does not satisfy the optimality relationship as KLD does [2]. Properly speaking, the SD is not a Karhunen-Loève decomposition. Finally, invariance with respect to linear transformation can also be deduced.

\subsection{Mechanical interpretation of $S D$}

As in the discrete case (see Section 2.3), the parameters of the SD (SMs and SVs) can be related to the normal modes (in terms of mode shapes and resonance frequencies) of a linear distributed-parameter system.

We consider here a linear distributed-parameter damped mechanical structure under random white-noise excitation. We assume that the motion is defined as

$$
\mathbf{u}(t, \mathbf{x})=\sum_{k=1}^{\infty} q_{k}(t) \Phi_{k}(\mathbf{x})
$$

where the family of functions $\Phi_{k}(\mathbf{x})$ coincides with the family of eigenfunctions (also named normal mode functions) of the eigenproblem associated to the equations of motion characterizing the motion of the undamped system [10]. In this case, the $q_{k}(t)$ are called normal coordinates. Assuming that the system is positive-define selfadjoint, the eigenproblem admits a denumerable infinite set of eigenvalues $\beta_{k}$ where $\omega_{k}=\sqrt{\beta_{k}}$ are the natural frequencies and the eigenfunctions satisfy the orthogonality property

$$
\int_{\mathcal{D}_{x}} m(x) \Phi_{k}(x)^{T} \Phi_{l}(x) d x=\delta_{k l}
$$

where $m(x)$ denotes the mass distribution.

The relationships between the SD parameters and the modal characteristics are based on the properties of the normal coordinates. If for the considered system, the normal coordinates $q_{k}$ satisfy uncoupled equations of motion [8]

$$
\ddot{q}_{k}(t)+2 \tau_{k} \omega_{k} \dot{q}_{k}(t)+\omega_{k}^{2} \ddot{q}_{k}(t)=p_{k}(t)
$$

with uncorrelated modal excitation components $p_{k}$, then the following relationships occur

$$
\nu_{k}=\frac{1}{\omega_{k}^{2}},
$$




$$
\Gamma_{k}(x)=m(x) \Phi_{k}(x)
$$

showing that the SVs $\left(\nu_{k}\right)$ of $\left\{\mathbf{u}(t, x),(t, x) \in \mathbb{R} \times \mathcal{D}_{x}\right\}$ are equal to the inverse of the square of the natural frequencies $\omega_{k}$ of the system and that the SMs $\left(\Gamma_{k}(x)\right)$ of $\left\{\mathbf{u}(t, x),(t, x) \in \mathbb{R} \times \mathcal{D}_{x}\right\}$ are simply related to the normal mode functions $\left(\Phi_{k}(x)\right)$ by the product with the mass distribution $m(x)$.

Substituting Eq. (20) into Eq. (17) reduces to

$$
\int_{\mathcal{D}_{x}} \Gamma_{k}(x) \Phi_{l}(x) d x=\delta_{k l}
$$

showing that the family of SMs are orthogonal to the family of the normal modes.

The relations Eqs (19) and (21) are the space-continuous versions of the relations Eq. (11) obtained for discrete mechanical systems. These relationships are only valid under proportional damping assumption and uncorrelated modal excitations.

\section{Illustrative example}

We consider a Euler-Bernoulli beam that is clamped at one end and free at the other. The model is the following

$$
\begin{aligned}
& \rho S \ddot{\mathbf{u}}(t, x)+E I \mathbf{u}^{I V}(t, x)=\delta\left(x-x_{f}\right) f(t) \\
& \mathbf{u}(t, 0)=0, \mathbf{u}^{I}(t, 0)=0 \\
& \mathbf{u}^{I I}(t, L)=0, \mathbf{u}^{I I}(t, L)=0
\end{aligned}
$$

with the usual parameters: $L$ the length, $S$ the cross-section area, $E$ the Young's modulus of elasticity, $I$ the moment of inertia, $\rho$ the mass density. We also assume that a space-localized (at $x=x_{f}$ ) excitation force, $f(t)$, is applied to the beam where $f(t)$ is a scalar random process with covariance function $\mathbb{E}\left(f(t+\tau) f^{T}(t)\right)=S_{f} \delta(\tau)\left(S_{f}>0\right)$.

Let $\left(\omega_{i}, \Phi_{i}\right)$ be the resonance frequencies and the modal functions associated to the unforced model Eq. (22). The displacement of the beam is approximated in a truncated series form

$$
\mathbf{u}(t, x)=\sum_{i=1}^{p} q_{i}(t) \Phi_{i}(x)
$$

where the dynamics of the modal components are given by the $p$ equations of motion

$$
\ddot{q}_{i}(t)+2 \tau \omega_{i} \dot{q}_{i}(t)+\omega_{i}^{2} q_{i}(t)=g_{i}(t)
$$

obtained applying a classical Galerkin procedure to Eq. (22) using the basis $\left(\Phi_{i}\right)_{i \geqslant 1}$. The modal excitation component $g_{i}$ is related to the physical excitation $f$ by

$$
g_{i}(t)=\Phi_{i}\left(x_{f}\right) f(t) .
$$

Note that the modal excitation components are correlated (the cross power spectral density function between $g_{i}$ and $g_{j}$ is given by $S_{f} \Phi_{i}\left(x_{f}\right) \Phi_{j}\left(x_{f}\right)$, for $1 \leqslant i, j \leqslant p$ ) showing that the SD approach can not give the exact modal parameters. As classical, proportional damping has been added to take into account dissipation.

For a given $p$, the covariance function $R_{\mathbf{u}}\left(0, x, x^{\prime}\right)$ was evaluated using the relation

$$
R_{\mathbf{u}}\left(0, x, x^{\prime}\right)=\sum_{i=1}^{p} \sum_{j=1}^{p} \Phi_{i}(x) \Phi_{j}\left(x^{\prime}\right) \mathbb{E}\left(q_{i}(t) q_{j}(t)\right)
$$

where $\mathbb{E}\left(q_{i}(t) q_{j}(t)\right)$ denotes the covariance quantities associated to the stationary response of the $p$ (for $\left.i=1, \ldots, p\right)$ coupled Eq. (24). The covariance matrix of Eq. (24) was obtained solving numerically the associated Lyapounov equation [2]. A similar approach was used to evaluate $R_{\dot{\mathbf{u}}}\left(0, x, x^{\prime}\right)$. 
Table 1

Resonance frequencies estimated from SD

\begin{tabular}{rccrrrrr}
\hline & SD & SD & SD & SD & \multicolumn{2}{c}{ SD } & \multicolumn{2}{c}{ SD } & Exact \\
& $N_{s}=2$ & $N_{s}=4$ & $N_{s}=6$ & \multicolumn{1}{c}{$N_{s}=8$} & $N_{s}=10$ & $N_{s}=20$ & \\
\hline$\omega_{1}$ & 4.58 & 4.57 & 4.57 & 4.57 & 4.57 & 4.57 & 4.57 \\
$\omega_{2}$ & 28.95 & 28.71 & 28.65 & 28.64 & 28.64 & 28.64 & 28.64 \\
$\omega_{3}$ & - & 81.83 & 80.48 & 80.26 & 80.21 & 80.18 & 80.18 \\
$\omega_{4}$ & - & 160.58 & 159.57 & 157.83 & 157.33 & 157.13 & 157.13 \\
$\omega_{5}$ & - & - & 270.37 & 263.34 & 260.86 & 259.80 & 259.75 \\
$\omega_{6}$ & - & - & 399.39 & 400.28 & 392.37 & 388.13 & 388.01 \\
$\omega_{7}$ & - & - & - & 572.29 & 555.32 & 542.42 & 541.94 \\
$\omega_{8}$ & - & - & - & 746.80 & 757.23 & 722.53 & 721.52 \\
$\omega_{9}$ & - & - & - & - & 988.56 & 929.23 & 926.75 \\
$\omega_{10}$ & - & - & - & - & 1199.40 & 1162.90 & 1157.60 \\
\hline
\end{tabular}

The SD method was then developed from a spatial sampling of the random field $\{\mathbf{u}(t, x),(t, x) \in \mathbb{R} \times[0, L]\}$.

Let $x_{k}=k \Delta x$ for $k=1, \ldots, N_{s}$ with $\Delta x=L / N_{s}$ and $N_{s}>1$ the $N_{s}$ sampling points. Starting from the sample functions, $R_{\mathbf{u}}\left(0, x_{i}, x_{j}\right)$ and $R_{\dot{\mathbf{u}}}\left(0, x_{i}, x_{j}\right)$, Eq. (13) can be written for $x=x_{i}$ given

$$
\int_{\mathcal{D}_{x}} R_{\mathbf{u}}\left(0, x_{i}, x^{\prime}\right) \Gamma_{k}\left(x^{\prime}\right) d x^{\prime}=\nu_{k} \int_{\mathcal{D}_{x}} R_{\dot{\mathbf{u}}}\left(0, x_{i}, x^{\prime}\right) \Gamma_{k}\left(x^{\prime}\right) d x^{\prime}
$$

Next, applying the numerical trapezoidal quadrature method to approximate the two integrals gives

$$
\sum_{j=1}^{N_{s}} \alpha_{j} R_{\mathbf{u}}\left(0, x_{i}, x_{j}\right) \Gamma_{k}\left(x_{j}\right)=\nu_{k}^{N_{s}} \sum_{j=1}^{N_{s}} \alpha_{j} R_{\dot{\mathbf{u}}}\left(0, x_{i}, x_{j}\right) \Gamma_{k}\left(x_{j}\right) .
$$

where $\alpha_{j}=1$ for $1<j<N_{s}-1$ and $\alpha_{N_{s}}=0.5$.

Finally, collecting for $i=1$ to $N_{s}$ the $N_{s}$ Eq. (27), the sample functions, $\Gamma_{k}\left(x_{j}\right)$, written as a vectors $\Gamma_{k}^{N_{s}}=$ $\left[\Gamma_{k}\left(x_{1}\right) \Gamma_{k}\left(x_{2}\right) \ldots \Gamma_{k}\left(x_{N_{s}}\right)\right]^{T}$ solves the eigenproblem

$$
\mathbf{A} \Gamma_{k}^{N_{s}}=\nu_{k}^{N_{s}} \mathbf{B} \Gamma_{k}^{N_{s}}
$$

where $\mathbf{A}$ and $\mathbf{B}$ are two $N_{s} \times N_{s}$ matrices which are easily obtained from Eq. (27).

Solving the eigenproblem Eq. (28) gives access to the SMs and to the SVs. The relation Eq. (19) can be used to approximate the resonance frequencies. The modal functions can be estimated using Eq. (21) which does not requires any information on the system.

Using the numerical trapezoidal quadrature method to approximate the integrals, Eq. (21) reduces to

$$
\sum_{j=1}^{N_{s}} \alpha_{j} \Gamma_{k}\left(x_{j}\right) \Psi_{l}\left(x_{j}\right)=\delta_{k l}
$$

Introducing the following notations

$$
\begin{aligned}
\tilde{\boldsymbol{\Gamma}}_{k}^{N_{s}} & =\left[\alpha_{1} \Gamma_{k}\left(x_{1}\right) \alpha_{2} \Gamma_{k}\left(x_{2}\right) \ldots \alpha_{N_{s}} \Gamma_{k}\left(x_{N_{s}}\right)\right]^{T} \\
\tilde{\boldsymbol{\Gamma}}^{N_{s}} & =\left[\tilde{\Gamma}_{1}^{N_{s}} \tilde{\Gamma}_{2}^{N_{s}} \ldots \tilde{\Gamma}_{N_{s}}^{N_{s}}\right] \\
\boldsymbol{\Phi}_{k}^{N_{s}} & =\left[\Phi_{k}\left(x_{1}\right) \Phi_{k}\left(x_{2}\right) \ldots \Phi_{k}\left(x_{N_{s}}\right)\right]^{T} \\
\boldsymbol{\Phi}^{N_{s}} & =\left[\boldsymbol{\Phi}_{1}^{N_{s}} \boldsymbol{\Phi}_{2}^{N_{s}} \ldots \boldsymbol{\Phi}_{N_{s}}^{N_{s}}\right]
\end{aligned}
$$

the $N_{s}^{2}$ Eq. (29) can then be written in the matrix form

$$
\boldsymbol{\Phi}^{N_{s}} \tilde{\boldsymbol{\Gamma}}^{N_{s}^{T}}=\boldsymbol{I}
$$



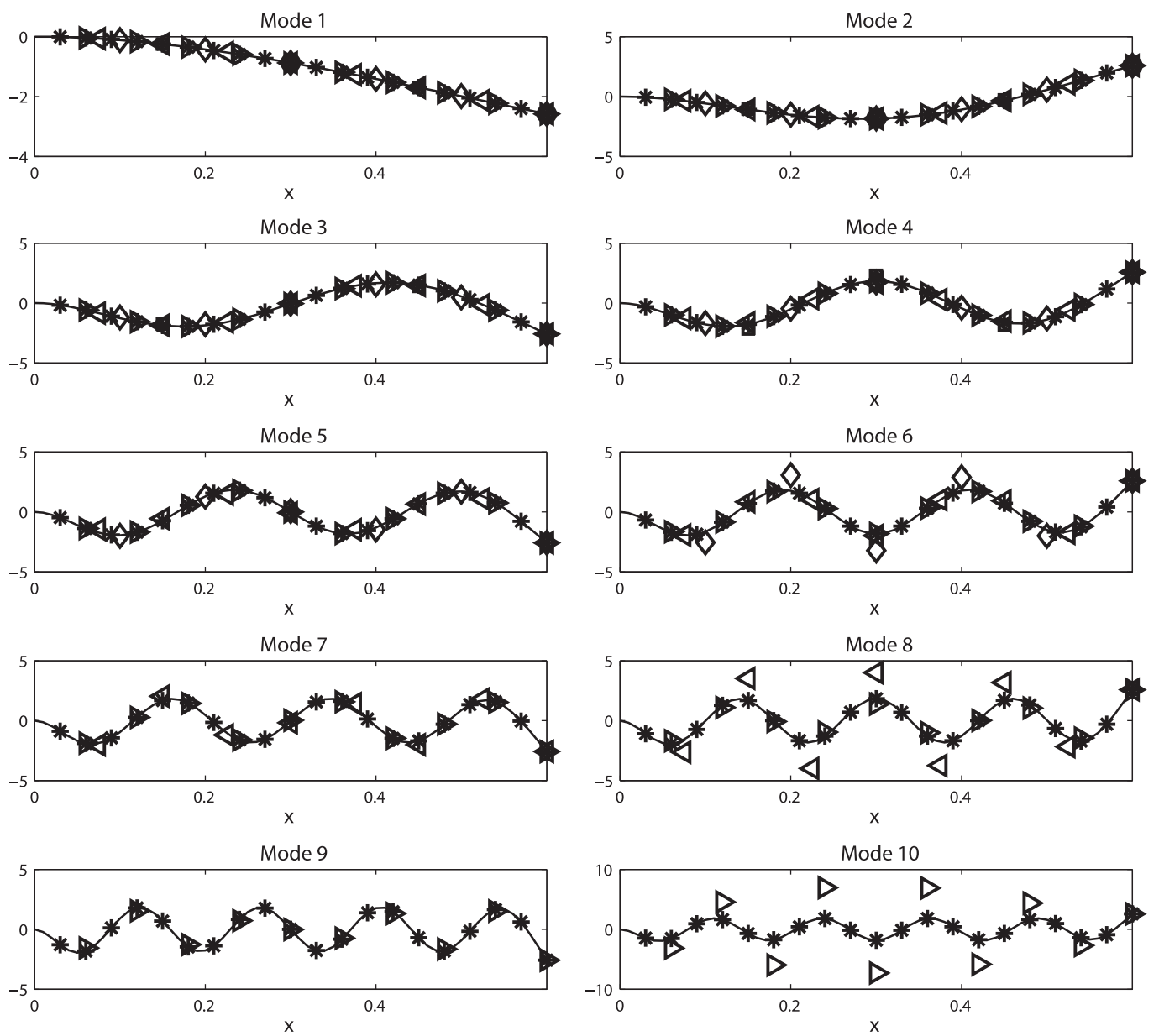

Fig. 1. The modal functions (solid line) and the modal functions approximated from the SD approach with $N_{s}=2(\circ), N_{s}=4$ ( $\square$ ), $N_{s}=6$ $(\diamond), N_{s}=8(\triangleleft), N_{s}=10(\triangleright)$ and $N_{s}=20(*)$.

where $\boldsymbol{I}$ denotes the $N_{s} \times N_{s}$ identity matrix given the relation

$$
\boldsymbol{\Phi}^{N_{s}}=\tilde{\boldsymbol{\Gamma}}^{N_{s}^{-T}}
$$

which has been used to estimate from the SMs the modal functions at the sampling points.

In Table 1, the exact first ten resonance frequencies of the clamped-free beam are compared with the associated ones obtained using the SD approach (Eq. (19)). The parameter values used are: $L=0.6, E I=1.4, \rho S=0.1620$, $\tau=0.005, x_{f}=0.05, S_{f}=1$ and $p=40$. The SD approach has been developed for $N_{s}=2,4,6,8,10$ and 20 sampling points. For $N_{s}=2$, the estimation of the first two resonance frequencies are not so bad. The accuracy increases quickly with $N_{s}$. For $N_{s}=10$, the first five frequencies are well estimated. Twenty points are needed to estimate satisfactorily the first ten frequencies.

In Fig. 1, the exact first ten modal functions of the clamped-free beam are compared with the associated ones obtained using the SD approach (Eq. (35)) with $N_{s}=2,4,6,8,10$ and 20 sampling points. With $N_{s}=2$ where only two modes are accessible, the SD approach gives values for the first two modal functions at the sampling points very closed to the exact one (see circle positions on the modes 1 and 2). The same comment is also valid for $N_{s}=4$. For the other values of $N_{s}\left(N_{s}=6,8\right.$ and 10) only the last accessible modal function is not correctly approximated. For $N_{s}=20$, the results illustrate well the numerical convergence with respect of the parameter $N_{s}$. 

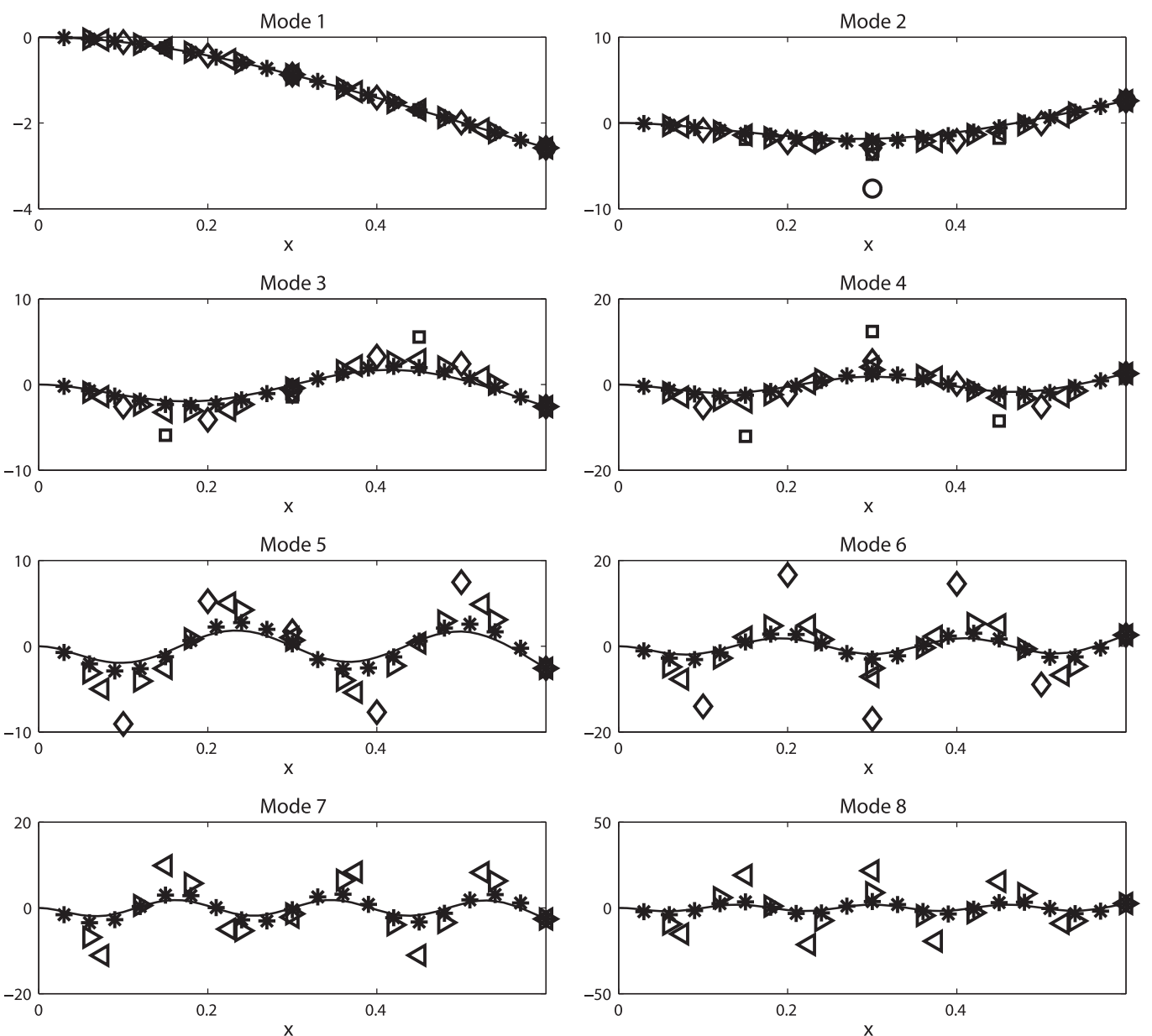

Fig. 2. The modal functions (solid line) and the KLMs with $N_{s}=2(\circ), N_{s}=4(\square), N_{s}=6(\diamond), N_{s}=8(\triangleleft), N_{s}=10(\triangleright)$ and $N_{s}=20(*)$.
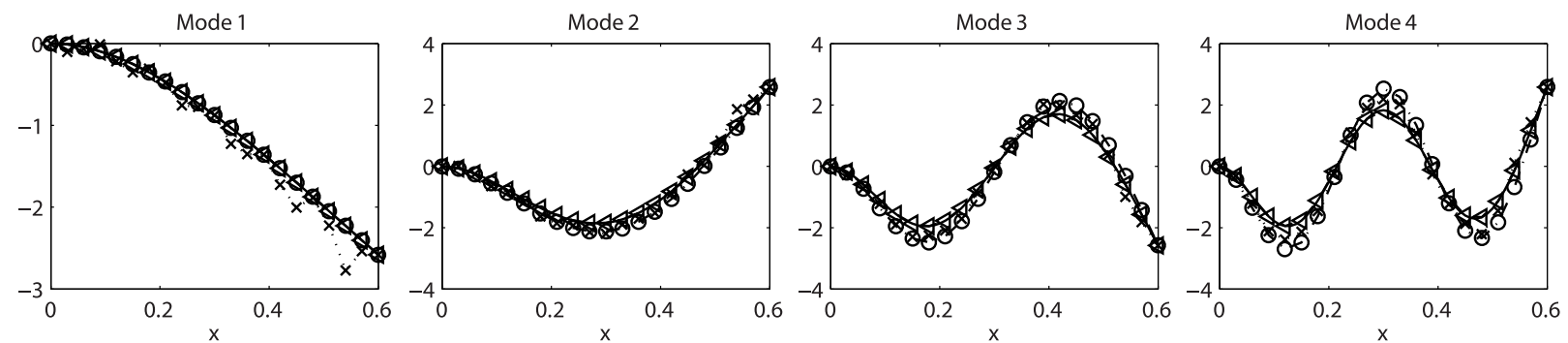

Fig. 3. The modal functions (solid line), the KLMs with $N_{s}=20$ (dotted-dashed line with o), the SMs with $N_{s}=20$ (dotted line with $\times$ ) and the modal functions approximated from the SD approach with $N_{s}=20$ (dashed line with $\triangleleft$ ).

In Fig. 2, the results obtained with the KLD approach using the same data are reported. When the number of sampling points is small, the KLMs show significant differences from the modal functions. Even for $N_{s}=20$, some differences are still present.

Finally, it is interesting to observe the SMs. They are plotted in Fig. 3 in case of $N_{s}=20$. As expected, they are very close to the modal functions but they show small spatial fluctuations. 


\section{Conclusion}

This paper has presented the potential of the smooth decomposition in terms of output-only modal analysis tool when stationary random responses are considered. The properties of the smooth decomposition when it is applied to a discrete linear mechanical system were recalled. The smooth decomposition was extended to the class of scalar stationary random processes indexed in time and space. It was shown that when the SD is applied to the stationary response of a linear distributed-parameter damped mechanical structure under random white-noise excitation, the same properties as in the discrete case hold. Under uncorrelated modal forcing terms, the SD gives access to resonance frequencies and modal functions. Simulations have shown that the SD approach can advantageously replace the KLD approach in the context of the modal analysis or in terms of dynamical analysis. Important but not analysis here is the dependency of the SMs with respect to the mass distribution which could be a tool to study the integrity of a structure and monitoring its healthy.

Current works include the use of the SD to analysis nonlinear problem and the develpment of a SD-based reduced order model.

\section{Acknowledgment}

The authors gratefully acknowledge the financial support of COFECUB, CAPES, Faperj, and CNPq.

\section{References}

[1] J.S.R. Anttonen, P.I. King and P.S. Beran, POM-based reduced-order model with deforming grids, Mathematical and Computer Modelling 38 (2003), 41-62.

[2] S. Bellizzi and R. Sampaio, POMs analysis of randomly vibrating systems obtained from Karhunen-Loève expansion, Journal of Sound and Vibration 297 (2006), 774-793.

[3] S. Bellizzi and R. Sampaio, Smooth Karhunen-Loève decomposition to analyze random vibrating systems, Journal of Sound and Vibration 325 (2009), 491-498.

[4] K. Carlberg and C. Farhat, A low-cost, goal-oriented compact proper orthogonal decomposition basis for model reduction of static systems, International Journal for Numerical methods in Engineering 86 (2011), 381-402.

[5] D. Chelidze and W. Zhou, Smooth orthogonal decomposition-based vibration mode identification, Journal of Sound and Vibration 292 (2006), 461-473.

[6] U. Farooq and B.F. Feeny, Smooth orthogonal decomposition for modal analysis of randomly excited systems, Journal of Sound and Vibration 316 (2008), 137-146.

[7] G. Kerschen, J.-C. Golinval, A. Vakakis and L. Bergman, The method of proper orthogonal decomposition for dynamical characterization and order reduction of mechanical systems: an overview, Non-linear Dynamics, Special Issue on Reduced Order Models: Methods and Applications Applications 41 (2005), 147-169.

[8] Y.K. Lin, Probability Theory of Structural Dynamics, McGraw-Hill, Inc, 1967.

[9] X. Ma, A.F. Vakakis and L. Bergman, Karhunen-Loève analysis and order reduction of the transient dynamics of linear coupled oscillators with strongly nonlinear end attachments, Journal of Sound and Vibration 309 (2008), 569-587.

[10] L. Meirovitch, Computational Method in Structural Dynamics, Sijthoff and Noordhoff International Publishers, 1980.

[11] R. Sampaio and S. Bellizzi, Analysis of non-stationary random processes using smooth decomposition, Journal of Mechanics of Materials and Structures 2011, (to appear). 

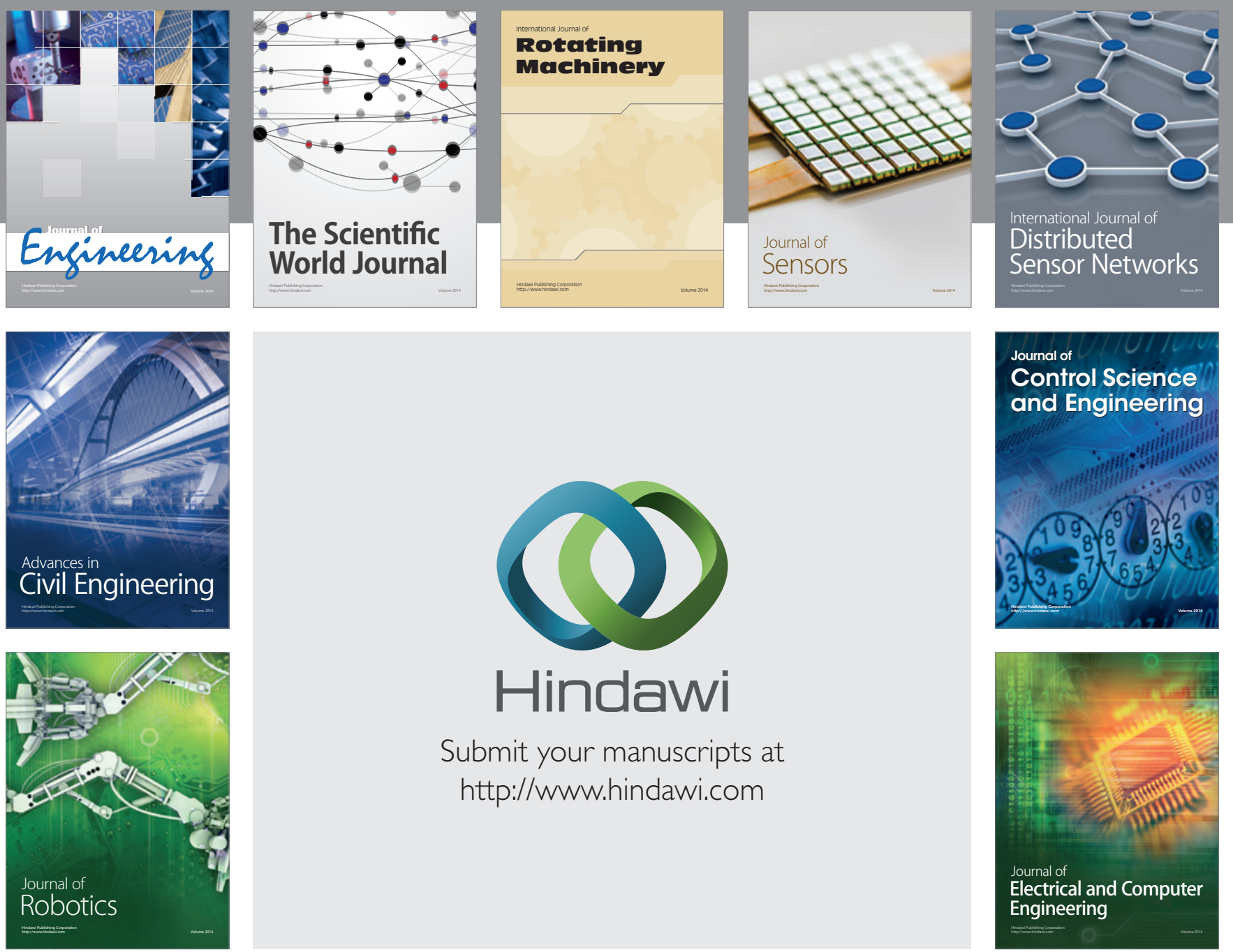

Submit your manuscripts at

http://www.hindawi.com
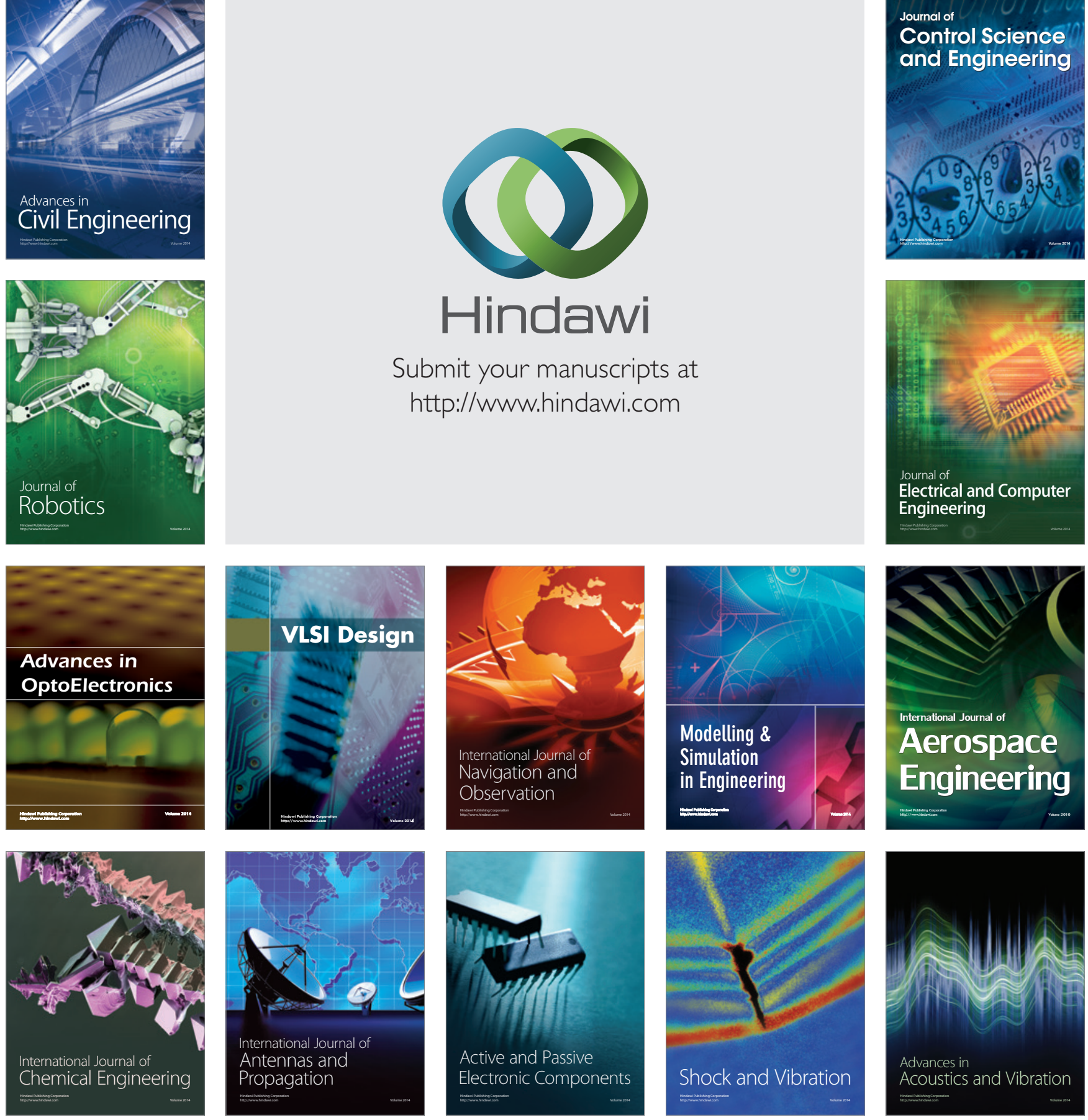